\title{
Interference Aware Resource Allocation with QoS Guarantees in OFDMA/SC-FDMA
}

\author{
Níbia Souza Bezerra, Tarcisio F. Maciel, F. Rafael M. Lima and Vicente A. Sousa Jr.
}

\begin{abstract}
Efficient Radio Resource Allocation (RRA) is of utmost importance for achieving maximum capacity in mobile networks. However, the performance assessment should take into account the main constraints of these networks. This letter presents important enhancements to RRA algorithms proposed in [1]. Prior work [1] ignores some important system constraints such as the impact of inter-cell interference and granularity of frequency allocation blocks. Here we show the performance degradation when these system constraints are assumed on the algorithms in [1] as well as propose some improvements on these algorithms in order to achieve better performance.
\end{abstract}

Index Terms-Radio resource allocation, Long Term Evolution, quality of service.

\section{INTRODUCTION}

$\mathbf{I}$ $\mathrm{N}$ [1], the authors formulate the RRA problem of spectral efficiency maximization subject to user satisfaction constraints in a multi-service wireless system. The nonlinear optimization problem is converted to an integer linear programming and the optimal solution is obtained by standard techniques. The high computational complexity of the optimal solution is managed by proposing a fast suboptimal algorithm named Reallocation-based Assignment for Improved Spectral Efficiency and Satisfaction (RAISES). RAISES represents a tradeoff between performance and computational complexity, obtaining near-optimal performance in low and medium loads for both Downlink (DL) and Uplink (UL) directions. However, the authors demonstrate the benefits of RAISES in a simple Long Term Evolution (LTE) single-cell modeling ignoring two important sources of performance degradation: inter-cell interference and Resource Block (RB) aggregation.

In this letter we revisit the RRA problem in [1] by including the inter-cell interference in the problem formulation. We also show the performance degradation on the original algorithms in [1] when inter-cell interference and RB aggregation is assumed. To overcome this performance loss, we propose modifications in the original algorithms in [1] in order to become more robust against inter-cell interference.

\section{SYSTEM MODELING IMPROVEMENTS}

Table I presents a list of all the sets and variables used along the text.

Níbia Souza Bezerra is with the Luleå University of Technology (e-mail: nibia.souza.bezerra@1tu.se).

Tarcisio F. Maciel and Fco. Rafael M. Lima are with the Federal University of Ceará (e-mail: \{rafaelm, maciel\}@gtel.ufc.br).

Vicente A. Sousa Jr. is with the Federal University of Rio Grande do Norte (e-mail: vicente.sousa@ct.ufrn.br).

The authors would like to thank FUNCAP, CAPES and CNPq for their financial and scholarship support.

Digital Object Identifier: 10.14209/jcis.2018.13
TABLE I

LIST OF VARIABLES AND SETS.

\begin{tabular}{|c|c|}
\hline$B$ & Number of LTE cells \\
\hline $\mathcal{S}$ & set of adjacent subcarriers \\
\hline$O$ & set of consecutive OFDM symbols in time \\
\hline$y$ & set of the indices of all possible RBs assignment patterns \\
\hline $\mathcal{Z}$ & set of services \\
\hline $\mathcal{B}$ & auxiliary UE set (initialized with the set of all UEs) \\
\hline$\widetilde{\Upsilon}$ & number of possible RBs assignment patterns \\
\hline $\mathcal{R}$ & the resource set of size $R$ \\
\hline $\mathcal{R}_{v}$ & set of RBs that compose the assignment pattern $v \in \mathcal{Y}$ \\
\hline$R$ & Number of RBs \\
\hline$M_{T}$ & Number of transmit antennas \\
\hline$M_{R}$ & Number of receive antennas \\
\hline$u$ & link of interest \\
\hline$i$ & receiver index \\
\hline$j$ & transmitter index \\
\hline $\mathbf{H}_{i, j}$ & $\begin{array}{l}\text { channel matrix for a link of interest } u \text {, between a receiver } \\
i \text { and a transmitter } j\end{array}$ \\
\hline$a$ & receiver antenna index \\
\hline$b$ & transmit antenna index \\
\hline$h_{a, b}$ & each element of $\mathbf{H}_{i, j}$ \\
\hline $\mathbf{M}_{j}$ & precoding transmit matrix \\
\hline $\mathbf{D}_{i}$ & decoding receive matrix \\
\hline $\mathbf{y}_{i}$ & prior-filtering received signal vector \\
\hline$\tilde{\mathbf{y}}_{i}$ & post-filtering received signal vector \\
\hline $\mathbf{x}_{j}$ & transmit signal vector sent from transmitter $j$ \\
\hline$\sigma_{i}$ & $M_{R} \times 1$ white ZMCSCG noise vector at the receiver $i$ \\
\hline$p_{u, l}$ & the power of stream $l$ of link $u$ \\
\hline $\mathbf{d}_{u, l}$ & decoding filter vector of stream $l$ of link $u$ \\
\hline $\mathbf{m}_{u, l}$ & precoding filter vector of stream $l$ of link $u$ \\
\hline$\tilde{\sigma}^{2}$ & filtered receive noise power \\
\hline $\mathbf{H}_{j}$ & $\begin{array}{l}\text { channel matrix between an interfering node } j \text { and a receiving } \\
\text { node } i\end{array}$ \\
\hline$\lambda$ & $\begin{array}{l}\mathrm{RB} \text { activity percentage that denotes the fraction of the RBs } \\
\text { that are in use on each interfering sector }\end{array}$ \\
\hline$\hat{P}_{S}^{U L}$ & peak transmission power for each UE on each subcarrier \\
\hline$P^{U L}$ & total terminal power \\
\hline$S$ & total number of subcarriers per RB \\
\hline$\alpha$ & $\begin{array}{l}\text { controlling parameter used to calculate the peak transmission } \\
\text { power }\end{array}$ \\
\hline$\tilde{\gamma}_{j, s, r}^{\mathrm{UL}}$ & SINR of the UE $j$ at the $s^{\text {th }}$ subcarrier of the RB $r$ \\
\hline$\alpha_{j}^{\mathrm{UL}}$ & $\begin{array}{l}\text { path loss plus shadowing of the link between UE } j \text { and its } \\
\text { serving sector }\end{array}$ \\
\hline$h_{j, s, r}^{\mathrm{UL}}$ & $\begin{array}{l}\text { channel transfer function for the } j^{\text {th }} \mathrm{UE} \text { at the } s^{\text {th }} \text { subcarrier } \\
\text { of the } r^{\text {th }} \mathrm{RB}\end{array}$ \\
\hline$\zeta$ & intra-cell interference experienced by UE $j$ \\
\hline$\xi$ & inter-cell interference experienced by UE $j$ \\
\hline$\left(\sigma^{s u b}\right)^{2}$ & $\begin{array}{l}\text { noise power at the receiver in the bandwidth of a single } \\
\text { subcarrier }\end{array}$ \\
\hline$\tilde{\gamma}_{j, v}^{\mathrm{OLMMSE}}$ & $\begin{array}{l}\text { effective SINR experienced by the data transmitted by UE } \\
j \text { with the RBs present in the assignment pattern } v \in \mathcal{y}\end{array}$ \\
\hline$q_{j, r}^{\mathrm{DL}}$ & DL transmit data rate of $\mathrm{UE} j$ in $\mathrm{RB} r$ \\
\hline$q_{j, v}^{\mathrm{UL}}$ & $\begin{array}{l}\text { UL transmit data rate of UE } j \text { when assigned to the } \\
\text { assignment pattern } v \in y\end{array}$ \\
\hline$k_{z}$ & $\begin{array}{l}\text { minimum required number of satisfied UEs of each service } \\
z\end{array}$ \\
\hline$q_{j, r}$ & rate of UEs $j$ at resource $r$ \\
\hline$t_{j}$ & target rate of UE $j$ in the current TTI \\
\hline
\end{tabular}


We model a multiuser LTE system composed by $B$ sectored cells in a tri-sectored site layout. Orthogonal Frequency Division Multiple Access (OFDMA) and Single Carrier Frequency Division Multiple Access (SC-FDMA) are modeled for DL and UL directions, respectively, as also single and multiple antenna schemes. The minimum available resource to be allocated to a UE is called RB. An RB is composed by a number adjacent subcarriers indexed by $s \in\{1, \cdots, \mathcal{S}\}$ in the frequency domain, and a number of consecutive OFDM symbols in time indexed by $o \in\{1, \cdots, O\}$, which correspond to a TTI. RBs are identified by the index $r \in\{1, \cdots, R\}$, which is determined by the system and subcarrier bandwidths.

Our propagation model includes a macrocell distancedependent path loss model, a log-normal shadowing component and the classical Independent and Identically Distributed ZMCSCG channel [2]. We consider a Multiple Input Multiple Output (MIMO) channel subject to interference with $B$ cochannel links, each of them with $M_{T}$ transmit and $M_{R}$ receive antennas. The channel matrix for a link of interest $u$, between a receiver $i$ and a transmitter $j$, is denoted by $\mathbf{H}_{i, j}$. It is an $M_{R} \times M_{T}$ matrix whose elements $h_{a, b}$ are the channel transfer function between the receiver antenna $a$ and transmit antenna $b$ of the MIMO link. We assume $h_{a, b}$ are obtained from the middle subcarrier among the $\mathcal{S}$ ones that compose a RB.

Before transmission, the signals sent through the link $u$ by the transmitter $j$ are precoded by a transmit matrix $\mathbf{M}_{j}$. The precoded signals traverse the channel $\mathbf{H}_{i, j}$ subjected to interference and noise and, at the receiver, are decoded by a receive matrix $\mathbf{D}_{i}$. Based on these definitions, the input-output relation for the MIMO channel for a certain link is given by

$\tilde{\mathbf{y}}_{i}=\mathbf{D}_{i} \mathbf{y}_{i}=\mathbf{D}_{i}\left(\mathbf{H}_{i, j} \mathbf{M}_{j} \mathbf{x}_{j}+\sum_{\forall k \neq j} \mathbf{H}_{i, k} \mathbf{M}_{k} \mathbf{x}_{k}+\boldsymbol{\sigma}_{i}\right)$,

where $\mathbf{y}_{i}$ and $\tilde{\mathbf{y}}_{i}$ are the prior-filtering received signal vector and the post-filtering received signal vector, respectively, $\mathbf{x}_{j}$ is the transmit signal vector sent from transmitter $j$ and $\sigma_{i}$ is the $M_{R} \times 1$ white ZMCSCG noise vector at the receiver $i$. We also assume that the channel of interest is perfectly known at the transmitter and receiver while the interference in the system may or may not be known at the receiver. Note that we have omitted the resource index $r$, and hereafter, we use index $u$ to represent the links of interest between a transmitter $j$ and a receiver $i$ for the sake of clarity. Therefore, we might refer to $\mathbf{H}_{i, j}, \mathbf{D}_{i}$, and $\mathbf{M}_{j}$ simply as $\mathbf{H}_{u}, \mathbf{D}_{u}$, and $\mathbf{M}_{u}$, respectively.

We assume Singular Value Decomposition (SVD) precoding which turns a MIMO channel into a set of decoupled Single Input Single Output (SISO) channels that do not interfere with each other [3]. The SVD of the channel matrix $\mathbf{H}_{u}=\mathbf{U}_{u} \Sigma_{u} \mathbf{V}_{u}^{H}$ provides unitary right and left singular vector disposed in the matrices $\mathbf{V}_{u}$ and $\mathbf{U}_{u}$, respectively. As such, precoding and decoding matrices are defined as $\mathbf{M}_{u}=\mathbf{V}_{u}$, and $\mathbf{D}_{u}=\mathbf{U}_{u}^{H}$.

To model inter-cell interference, we can measure the channel quality for a spatial stream by means of its SINR, written as

$$
\tilde{\gamma}_{u, l}=\underbrace{\sum_{m \neq l} p_{u, m}\left|\mathbf{d}_{u, l} \cdot \mathbf{H}_{u} \cdot \mathbf{m}_{u, m}\right|^{2}}_{\text {intra-cell interference }}+\underbrace{\sum_{j \neq u} \sum_{n} p_{j, n}\left|\mathbf{d}_{u, l} \cdot \mathbf{H}_{j} \cdot \mathbf{m}_{j, n}\right|^{2}}_{\text {inter-cell interference }}+\underbrace{\tilde{\sigma}^{2}}_{\text {noise }},
$$

where $p_{u, l}$ is the power of stream $l$ of link $u, \mathbf{d}_{u, l}$ is the decoding filter vector of stream $l$ of link $u, \mathbf{m}_{u, l}$ is the precoding filter vector of stream $l$ of link $u, \tilde{\sigma}^{2}$ is the filtered receive noise power and $\mathbf{H}_{j}$ is the channel matrix between an interfering node $j$ and a receiving node $i$.

In order to model the level of inter-cell interference we assume $\lambda$ as the RB activity percentage that denotes the fraction of the RBs that are in use on each interfering sector. For example, if we set $\lambda$ parameter in $50 \%$, each interfering cell sector will randomly select approximately $50 \%$ of the RBs to cause inter-cell interference on interest cell sector.

In this letter, we also propose to evaluate the impact of resource allocation granularity on RAISES performance by means of the RB aggregation, which it is a practical limitation of LTE systems. It arranges the schedulable RBs in groups called Resource Block Group (RBG) [4], with the size depending on the system bandwidth. We assume the resource allocation type 0 in which $\mathrm{RB}$ allocation granularity (RBG size) is defined according to Table II.

TABLE II

TyPe 0 Resource Allocation On DL.

\begin{tabular}{c|c|c|c|c}
\hline \# of RBs $\left(N_{R B}^{D L}\right)$ & $\leqslant 10$ & $11-26$ & $27-64$ & $65-110$ \\
\hline RBG Size (in RBs) & 1 & 2 & 3 & 4 \\
\hline
\end{tabular}

Due to SC-FDMA in UL, RBs are contiguously allocated to the UEs in the form of virtual resource blocks [4]. According to [5], the number $\Upsilon$ of possible assignment patterns is given by $\Upsilon=(1 / 2) R^{2}+(1 / 2) R+1$, from which we can define $\mathcal{Y}=$ $\{1, \cdots, \Upsilon\}$ as the set of the indices of all possible assignment patterns. For a case with $R=4$ and $\Upsilon=11$, we can write a binary RB allocation matrix of dimension $R \times \Upsilon$ as

$$
\boldsymbol{\Omega}=\left[\begin{array}{lllllllllll}
0 & 1 & 0 & 0 & 0 & 1 & 0 & 0 & 1 & 0 & 1 \\
0 & 0 & 1 & 0 & 0 & 1 & 1 & 0 & 1 & 1 & 1 \\
0 & 0 & 0 & 1 & 0 & 0 & 1 & 1 & 1 & 1 & 1 \\
0 & 0 & 0 & 0 & 1 & 0 & 0 & 1 & 0 & 1 & 1
\end{array}\right],
$$

where each $\omega_{r, v}$ matrix element is set to 1 , when an $\mathrm{RB}$ is assigned to a specific assignment pattern, and 0 otherwise.

Additionally, according to [5], besides the resource allocation restrictions, we should respect the peak transmission power for each UE on each subcarrier $\left(\hat{P}_{S}^{U L}\right)$ as

$$
\hat{P}_{S}^{U L}=\frac{P^{U L}}{(\alpha * S)},
$$

where $P^{U L}$ is the total terminal power, $S$ is the total number of subcarriers per $\mathrm{RB}$, and $\alpha$ is a controlling parameter. Then, the power each UE can use to transmit in a subcarrier is

$$
\check{p}_{S}^{U L}=\min \left(\frac{P^{U L}}{S \times R}, \hat{P}_{S}^{U L}\right) .
$$

Now, we can calculate the SINR of the UE $j$ at the $s^{\text {th }}$ subcarrier of the RB $r$ as 


$$
\tilde{\gamma}_{j, s, r}^{\mathrm{UL}}=\frac{\underbrace{\zeta}_{\text {intra-cell interference }}+\underbrace{\check{p}_{s}^{U L} \cdot \alpha_{j}^{\mathrm{UL}} \cdot\left|h_{j, s, r}^{\mathrm{UL}}\right|^{2}}_{\text {inter-cell interference }}+\left(\sigma^{s u b}\right)^{2}}{\xi}
$$

where $\alpha_{j}^{\mathrm{UL}}$ is the path loss plus shadowing of the link between $\mathrm{UE} j$ and its serving sector, $h_{j, s, r}^{\mathrm{UL}}$ is the channel transfer function for the $j^{\text {th }} \mathrm{UE}$ at the $s^{\text {th }}$ subcarrier of the $r^{\text {th }} \mathrm{RB}$, $\zeta$ and $\xi$ represent the intra-cell and the inter-cell interference experienced by $\mathrm{UE} j$, respectively, and $\left(\sigma^{s u b}\right)^{2}$ is the noise power at the receiver in the bandwidth of a single subcarrier.

We also define the channel in function of subcarriers, instead of RBs, due to the frequency domain equalization of SCFDMA systems [6]. As in [1], we use a Minimum Mean Square Error (MMSE) equalizer, from which we can calculate the SINR of the data delivered by a certain RB set in a scenario with inter-cell interference as

$$
\tilde{\gamma}_{j, v}^{\mathrm{UL} M M S E}=\left(\frac{1}{\frac{1}{c \cdot\left|\mathcal{R}_{v}\right|} \sum_{r \in \mathcal{R}_{v}} \sum_{z=1}^{S} \frac{\tilde{\gamma}_{j, z, r}^{\mathrm{UL}}}{\tilde{\gamma}_{j, z, r}^{\mathrm{UL}}+1}}-1\right)^{-1},
$$

where $\tilde{\gamma}_{j, v}^{\mathrm{UL} \text { MMSE }}$ is the effective SINR experienced by the data transmitted by UE $j$ with the RBs present in the assignment pattern $v \in \mathcal{Y}$, and $\mathcal{R}_{v}$ is the set of RBs that compose the assignment pattern $v$.

Finally, we introduce a link adaptation function responsible for mapping the SINR to the transmission data rate as well as to model data block error. We assume a set of Modulation and Coding Schemess (MCSs), each one with different performance regarding the Block Error Rate (BLER). The DL BLER performance curves come from [7]. For UL, we resorted to the simulator available in [8], with which we obtained BLER curves for 15 different MCSs.

\section{CRM ALGORITHM IMPROVEMENTS}

Reviewing the problem formulated in [1] named Constrained Rate Maximization (CRM), we propose the inclusion of inter-cell interference in the optimization problem. For DL, the transmit data rate of $\mathrm{UE} j$ in $\mathrm{RB} r$ is now defined as

$$
q_{j, r}^{\mathrm{DL}}= \begin{cases}\sum_{l}^{c_{j}} f\left(\gamma_{l}\right), & \text { without interference awareness } \\ c_{j} & \\ \sum_{l} f\left(\tilde{\gamma}_{l}\right), & \text { with interference awareness }\end{cases}
$$

where $\gamma$ and $\tilde{\gamma}$ are the Signal-to-Noise Ratio (SNR) and SINR, respectively, $c_{j}$ denotes the number of transmitted signals (or streams), i.e. $c_{j} \leq \min \left(M_{T}, M_{R}, v\right)$, with $v$ being the rank of the $\mathbf{H}_{i, j} \cdot f(\cdot)$ is a link adaptation function, responsible for mapping the SNR/SINR to the transmit data rate and, in order to change the transmit data rate, we assume that the modulation and channel coding rates are changed according to the channel state. We assume that there are some MCS, each one with different performance regarding the BLER. Our link adaptation is performed based on the Channel Quality Indicator (CQI) table presented in [4]. The resource index $r$ was removed for sake of notation simplicity.
We define $\mathbf{X}^{\mathrm{DL}}$ as a $J \times R$ assignment matrix with elements $x_{j, r}^{\mathrm{DL}}$ that are valued as 1 if the $\mathrm{RB} r \in \mathcal{R}$ is assigned to the UE $j$, and 0 otherwise. We can also define $t_{j}$ as the data rate requirement from UE $j$ in the current TTI, $\mathcal{Z}$ as the set of services ${ }^{1}$ in the system, where $k_{z}$ is the minimum number of UEs from service $z \in \mathcal{Z}$ that must be satisfied. Then, we can now rewrite the DL CRM problem in its standard form as

$$
\max _{\mathbf{X}^{\mathrm{DL}}}\left(\sum_{j \in \mathcal{J}} \sum_{r \in \mathcal{R}} q_{j, r}^{\mathrm{DL}} \cdot x_{j, r}^{\mathrm{DL}}\right),
$$

subject to

$$
\begin{aligned}
& \sum_{j \in \mathcal{J}} x_{j, r}^{\mathrm{DL}}=1, \forall r \in \mathcal{R}, \\
& \sum_{j \in \mathcal{J}_{z}} u\left(\sum_{r \in \mathcal{R}} q_{j, r}^{\mathrm{DL}} \cdot x_{j, r}^{\mathrm{DL}}, t_{j}\right) \geq k_{z}, \forall z \in \mathcal{Z},
\end{aligned}
$$

where $u(a, b)$ is a step function that assumes the value 1 with $a \geq b$ and 0 otherwise, and $\mathcal{J}_{z}$ is the set of UEs that belongs to service $z$.

For UL, the transmit data rate of UE $j$ when assigned to the assignment pattern $v$ is defined as

$$
q_{j, v}^{\mathrm{UL}}= \begin{cases}f\left(\gamma_{j, v}^{\mathrm{UL} M M S E}\right), & \text { without interference awareness } \\ f\left(\tilde{\gamma}_{j, v}^{\mathrm{ULMMSE}}\right), & \text { with interference awareness }\end{cases}
$$

As in DL, we can now write the UL CRM problem in its standard form as

$$
\max _{\mathbf{X}^{\mathrm{UL}}}\left(\sum_{j \in \mathcal{J}} \sum_{v \in \mathcal{Y}} q_{j, v}^{\mathrm{UL}} \cdot x_{j, v}^{\mathrm{UL}}\right),
$$

subject to

$$
\begin{aligned}
& \sum_{j \in \mathcal{J}} \sum_{v \in \mathcal{Y}} \omega_{r, v} \cdot x_{j, v}^{\mathrm{UL}}=1, \quad \forall r \in \mathcal{R}, \\
& \sum_{v \in \mathcal{Y}} x_{j, v}^{\mathrm{UL}}=1, \quad \forall j \in \mathcal{J}, \\
& \sum_{j \in \mathcal{J}_{\mathcal{Z}}} u\left(\sum_{v \in \mathcal{Y}} q_{j, v}^{\mathrm{UL}} \cdot x_{j, v}^{\mathrm{UL}}, t_{j}\right) \geqslant k_{z}, \quad \forall z \in \mathcal{Z} .
\end{aligned}
$$

In this letter, equations (9a) and (11a) (objective functions) as well as Equations (9c) and (11d) (minimum number of satisfied UEs for each service) are now written as a function of equations (8) and (10).

Besides the modification on CRM problem, we provide changes in RAISES solution. Originally, RAISES is divided in two parts: (i) Unconstrained Maximization, which allocates resources to achieve the highest system data rate; and (ii) Reallocation, which reallocates RBs from the UEs with better channel condition (donors) to the ones with worst situation (receivers), trying to assure a pre-defined target UE data rate. As depicted in Figure 1, we propose new steps (the second and the third ones) to Unconstrained Maximization phase in order to provide RAISES to be also interference-aware. Then, we maintain the original idea to maximize the data rate, but now if the algorithm is aware of interference (step (2)), then

\footnotetext{
${ }^{1}$ We assume that the UEs can be grouped according to the multimedia data service used. Examples of data services are web browsing and video streaming.
} 
in step (3), the maximum rate allocation with the interference knowledge is performed.

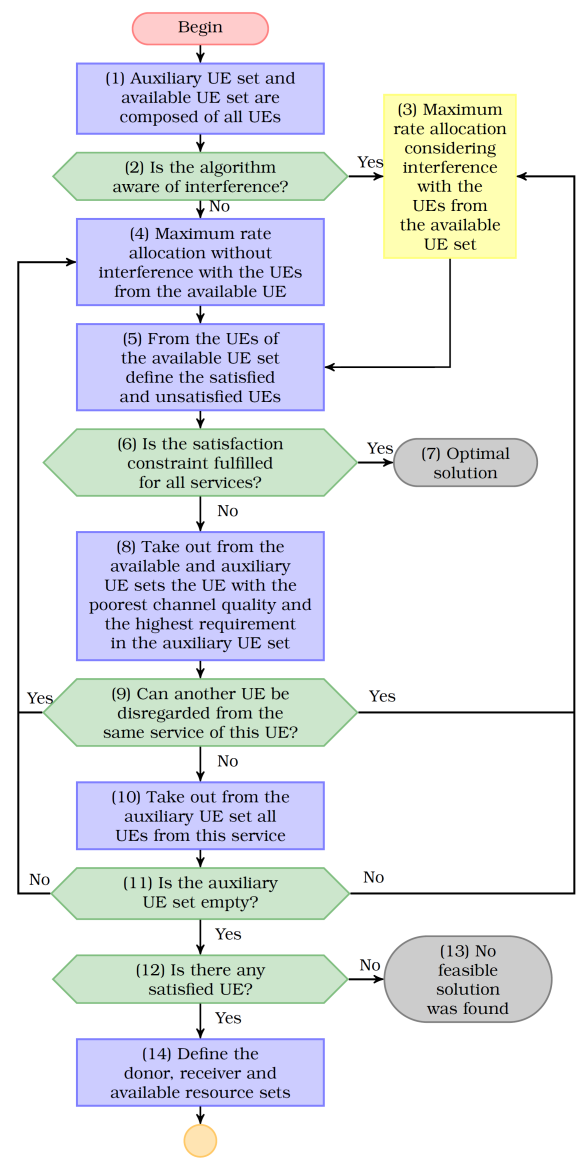

Fig. 1. Flowchart of the Unconstrained Maximization for DL.

As in original Unconstrained Maximization part of RAISES, if the minimum required number $k_{z}$ of satisfied UEs of each service $z$ is not achieved, a UE will not receive resources at the current TTI, thus it will be ignored. The criterion to select the UE $j^{\star}$ that will not receive any resources in DL and UL is given by

$$
j_{D L}^{\star}=\arg \min _{j \in \mathcal{B}} \frac{\left(\frac{1}{R} \sum_{r \in \mathcal{R}} q_{j, r}\right)}{t_{j}}, \quad j_{U L}^{*}=\arg \min _{j \in \mathcal{B}} \frac{\frac{1}{S \cdot R} \sum_{r \in \mathcal{R}} \sum_{z=1}^{S} \tilde{\gamma}_{j, z, r}^{\mathrm{UL}}}{q_{j}},
$$

where $\mathcal{B}$ is the auxiliary UE set (initialized with the set of all UEs), $\mathcal{R}$ is the resource set of size $R, q_{j, r}$ is the rate of UEs $j$ at resource $r$, and $t_{j}$ is the target rate of UE $j$ in the current TTI. With this criterion, the sets of donors and receivers UEs are defined. We follow the original of Reallocation part, which the basic idea consists in reallocating RBs from the donors to the receivers.

\section{PERformance Evaluation}

We evaluate the suboptimal solution of the CRM problem for a system with two different services whose UEs have the same rate requirement. Full buffer traffic model, SISO and 2x2 MIMO antenna arrangements are tested for both services.

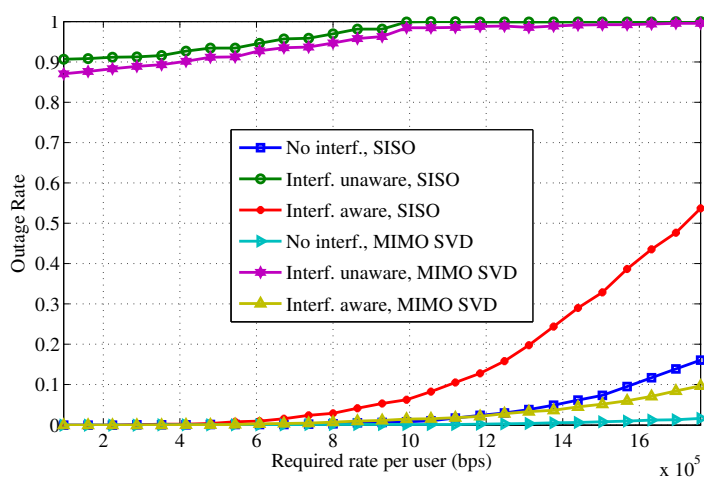

Fig. 2. DL Outage rate for SISO and MIMO (original versus modified RAISES).

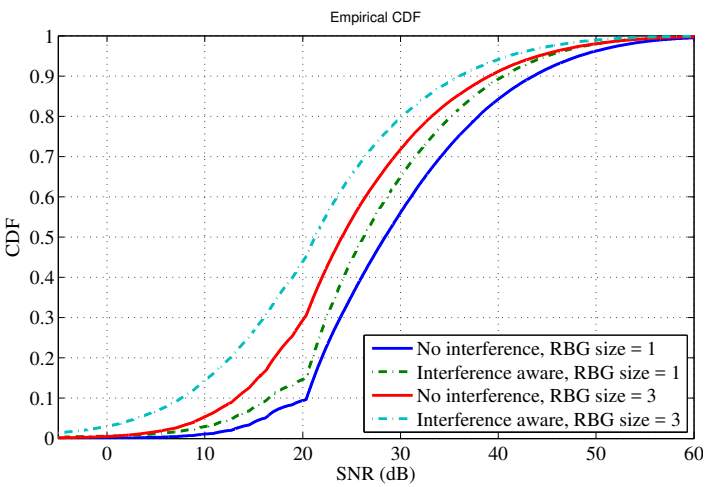

Fig. 3. DL SINR for SISO with $45 \mathrm{RBs}$ and $50 \%$ of cell loading. Required rate: $800 \mathrm{kbps}$.

Figure 2 presents the DL outage rate in a scenario with 45 available RBs with a cell loading $(\lambda)$ of $50 \%$. An outage event happens when RAISES can not find a feasible solution. Then, the outage rate is defined as the ratio between the number of snapshots with outage events and the total number of simulated snapshots (3000). As expected, MIMO provides better performance by taking advantage of spatial dimension. Also, the deployment of the original RAISES in a scenario with intercell interference (Interf. unaware case) provides outage rates higher than $90 \%$ for all antenna arrangements. However, the proposed modifications on RAISES (Interf. aware case) significantly approach its performance when subject to interference to the case without interference (No Interf. case), especially with MIMO. This evidences that the knowledge of interference can considerably increase the RAISES performance.

Figure 3 presents the impact of RB aggregation on CDFs of the SINR for SISO. Looking at the $50^{\text {th }}$ percentile, there is a difference of $18.33 \%$ between the case with and without $\mathrm{RB}$ aggregation when the interference is not modeled, and a corresponding difference of $23.58 \%$ for the case where RAISES is aware of interference. These differences occur due to the loss in granularity of resource assignment caused by $\mathrm{RB}$ aggregation. With RBG size equals to 3 , RAISES has to allocate in blocks of $3 \mathrm{RBs}$, instead of assigning each RB at a time.

Figure 4 shows the outage rates for UL direction. As with DL, the knowledge of inter-cell interference in the UL leads 


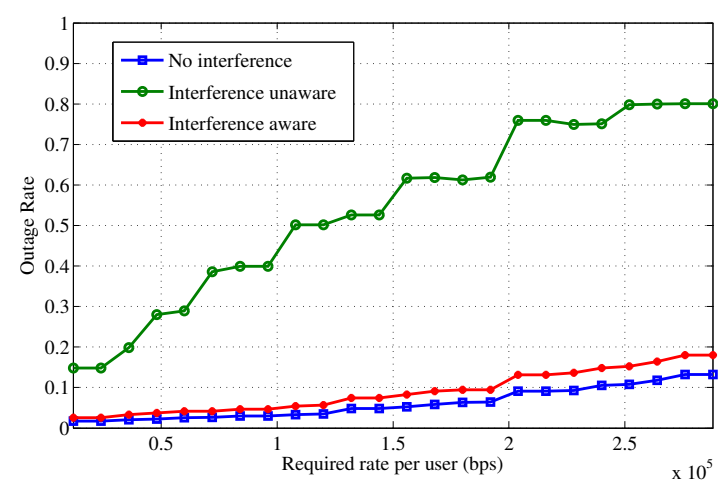

Fig. 4. Comparison of the outage rates for the three scenarios, $\lambda=100 \%$, $\alpha=5$.

to significant gains compared to interference unaware case. The knowledge of interference at the transmitter allows for better estimative of the channel quality and consequently a more precise resource allocation.

\section{CONCLUSION}

RRA algorithms are responsible for the assignment of frequency/time/power resources in order to guarantee Quality of Service (QoS) metrics at acceptable levels, allowing the UEs to have their satisfaction constraints fulfilled. Even using a combination of different techniques to achieve those goals, interference in multi-cellular networks can cause significant performance degradation. To measure and overcome this negative impact, we presented the evaluation of the suboptimal algorithm of RRA proposed in [1] in a scenario subjected to inter-cell interference and to some practical limitations of LTE systems such as RB aggregation. Results indicates that the proposed modified algorithm is almost capable of recovering the whole performance loss of original algorithm in [1] due to inter-cell interference degradation.

\section{REFERENCES}

[1] F. R. M. Lima, T. F. Maciel, W. C. Freitas, and F. R. P. Cavalcanti, "Resource Assignment for Rate Maximization with QoS Guarantees in Multiservice Wireless Systems," IEEE Transactions on Vehicular Technology, vol. 61, no. 3, pp. 1318-1332, March 2012, doi: 10.1109/TVT.2012.2183905.

[2] A. Paulraj, R. Nabar, and D. Gore, Introduction to Space-Time Wireless Communications, 1st ed. Cambridge University Press, 2003.

[3] A. Paulraj, E. Biglieri, and A. Goldsmith, MIMO Wireless Communications, 1st ed. Cambridge University Press, 2007.

[4] 3GPP, "Evolved Universal Terrestrial Radio Access (E-UTRA); Physical layer procedures," 3rd Generation Partnership Project (3GPP), TS 36.213, Oct. 2010. [Online]. Available: http://www.3gpp.org/ftp/Specs/htmlinfo/36213.htm

[5] I. C. Wong, O. Oteri, and W. Mccoy, "Optimal Resource Allocation in Uplink SC-FDMA Systems," IEEE Transactions on Wireless Communications, vol. 8, no. 5, pp. 2161-2165, May 2009, doi: 10.1109/TWC.2009.061038.

[6] H. G. Myung, J. Lim, and D. J. Goodman, "Single Carrier FDMA for Uplink Wireless Transmission," IEEE Vehicular Technology Magazine, vol. 1, no. 3, pp. 30-38, Sep. 2006, doi: 10.1109/MVT.2006.307304.

[7] C. Mehlführer, M. Wrulich, J. C. Ikuno, D. Bosanska, and M. Rupp, "Simulating the Long Term Evolution Physical Layer," in European Signal Processing Conference, Glasgow, Scotland, Aug. 2009.

[8] LTE Uplink Link Level Simulator. [Online]. Available: http://bit.ly/1910WFR

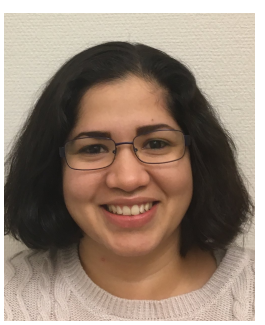

Níbia Souza Bezerra received the B.Sc. degree in Computer Engineering from Amazonas State University in 2008 and her M.Sc. in Teleinformatics Engineering from the Federal University of Ceará in 2011. From 2008 to 2011 she worked for Nokia Technology Institute (INdT) as a researcher, doing research related to wireless communications. From 2011 to 2013 she was a researcher at the Wireless Telecom Research Group (GTEL), Fortaleza, Brazil, where she worked in projects in cooperation with Ericsson Research. She is currently pursuing her $\mathrm{Ph} . \mathrm{D}$. in Computer Sciences at the Luleå University of Technology, in cooperation with Ericsson AB in Luleå, Sweden. Her research interests include mobility for machine-type communication, smart cities, and Internet of Things (IoT) for current wireless networks and future $5 \mathrm{G}$.

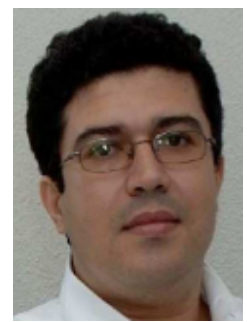

Tarcisio F. Maciel received his B.Sc. and M.Sc. degrees in Electrical Engineering from the Federal University of Ceará (UFC) in 2002 and 2004, respectively, and his Dr.-Ing. degree from the Technische Universität Darmstadt (TUD), Germany, in 2008, also in Electrical Engineering. Since 2001, he has actively participated in several projects in a technical and scientific cooperation between Wireless Telecom Research Group (GTEL), UFC, and Ericsson Research. From 2005 to 2008, he was a research assistant with the Communications Engineering Laboratory, TUD. Since 2008, he has been a member of the PostGraduation Program in Teleinformatics Engineering, UFC. In 2009, he was a Professor of computer engineering with UFC-Sobral and since 2010, he has been a Professor with the Center of Technology, UFC. His research interests include radio resource management, numerical optimization, and multiuser/multiantenna communications.

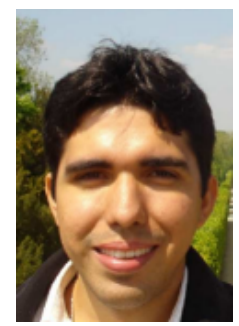

F. Rafael M. Lima received the B.Sc. degree with honors in Electrical Engineering in 2005, and M.Sc. and D.Sc. degrees in Telecommunications Engineering from the Federal University of Ceará, Fortaleza, Brazil, in 2008 and 2012, respectively. In 2008, he has been in an internship at Ericsson Research in Luleå, Sweden, where he studied scheduling algorithms for LTE system. Since 2010, he has been a Professor of Computer Engineering Department of Federal University of Ceará, Sobral, Brazil. Prof. Lima is also a researcher at the Wireless Telecom Research Group (GTEL), Fortaleza, Brazil, where he works in projects in cooperation with Ericsson Research. In 2016, prof. Lima has been awarded a research productivity scholarship by FUNCAP. He has published several conference and journal articles as well as patents in the wireless telecommunications field. His research interests include radio resource allocation algorithms for QoS guarantees in scenarios with multiple services, resources, antennas, and users.

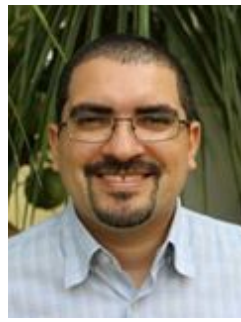

Vicente A. Sousa Jr received his B.Sc., M.Sc., and $\mathrm{Ph} . \mathrm{D}$. degrees in electrical engineering from Federal University of Ceará (UFC) in 2001, 2002, and 2009, respectively. Between 2001 and 2006, he developed solutions to UMTS/WLAN interworking for UFC and Ericsson of Brazil. Between 2006 and 2010, he contributed to WIMAX standardization and Nokia's product as a researcher at Nokia Technology Institute (INdT). He is now a lecturer at Federal University of Rio Grande do Norte (UFRN), Brazil. 